THE NUMERTCAL DESIGN OF FEEDBACK CONTROL SYSTEMS CONTA INING A SATURATTON ELEMENT BY THE METHOD OF INEQUALITTES

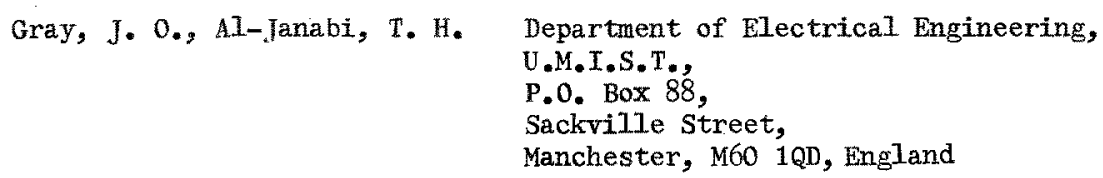

This paper is concerned with the numerical synthesis of compensators for single loop feedback control systems containing a single non-linear element of the saturation type. The approach adopted is that of the method of inequalities (1) where the problem is formulated by a set of inequalities which represent the closed loop performance required from the system as well as constraints of a physical engineering or financial kind. The inequalities define a set of points called the admissible space in the space of possible controller parameters and any point in this set represents an acceptable design. The approach differs from that of optimisation in that system performance is specified by a set of inequalities rather than a cost function, so that every aspect of the desired performance can be independently specified.

In previous work $(1)$ the method was applied to the design of linear systems and here the method is extended to non-linear systems by the addition of a new inequality which ensures that every point in the admissible space represents a closed loop system which does not exhibit autonomous limit cycle oscillation. This inequlity is defined by using an enhanced sinusoidal describing function algorithm recently proposed by Mees ${ }^{(2)}$ which takes account of the effects of higher harmonic signal. components as well as the fundamental sinusoid in the search for regions of possible limit cycle operation. To simplify the numerical calculation of indicial responses, the non-linear element is replaced by an equivalent linear gain using the technique of the exponential describing function. (3) An example of use is given and the method evaluated.

\title{
2. The design method
}

The method of inequalities represents a completely general design philosophy which can be used to encompass constraints in both the time and frequency domain. In the extension to non linear systems it is simply required to find suitable representations for the non linear elements, a choice which will be determined by the way in which the closed loop specifications are to be formulated. For convenience the method of inequalities is now briefly reviewed and the algorithms used in this work outlined.

\subsection{The method of inequalities}

A design problem can be formulated in terms of a set of inequalities.

$$
\phi_{i}(\mathrm{p}) \leqslant \mathrm{C}_{\mathrm{i}}, \quad \mathrm{i}=1,2 \ldots \ldots \ldots+\mathrm{m}-\text { (1 }
$$

where the $\mathrm{C}_{i}$ are real numbers and $p$ denotes the real vector $\left[p_{1}, \ldots . \cdots p_{n}\right]$ and $\phi_{i}$ are real functions of $p$. The inequalities (1) can represent performance specifications and system constraints which can be of a physical, engineering or financial kind and the components of $p$ 
represent system parameters. Each inequality

$\phi(p) \leqslant c_{i}$ defines a set of $s_{i}$ of points in the $n$ dimensional space $\mathrm{R}^{\mathrm{n}}$; the coordinates of this space are $\mathrm{p}_{1}, \mathrm{p}_{2} \ldots \ldots . \mathrm{p}_{\mathrm{n}}$. We can write

$$
s_{i}=\left\{p: \phi_{i}(p) \quad c_{i} \quad--(2\right.
$$

where the boundary of $s_{i}$ is defined by $\phi_{i}(p)=c_{i}$.

If there is a point $p$ in $\mathrm{R}^{\mathrm{n}}$ that satisfies simultaneously all the inequalities

$f_{i}(p) \leqslant c_{i} \quad i=1,2 \ldots . . m$ then $p$ is inside every set $s_{i}$. Let $s$ denote the intersection of all sets $s_{i}$; in the usual notation

$$
s=\bigcap_{i=1}^{m} s_{i}
$$

Thus $p$ satisfies all the inequalities if and only if $p$ is in $s$. We say that $S$ is an admissable set and any $p$ in $S$ is an admissable point. This concept is illustrated graphically in Figure 1.

Computer programming techniques have been developed for the determination of controllers for single variable and multivariable linear systems using this design philosophy where the $\not b$ (p) relate to desired constraints on the loop indicial responses and loop interaction effects and also to practical constraints on such factors as controller parameter values and the magnitude of forcing functions. The direct extension of these techniques to non linear systems required the use of an algorithm to represent the non linear element. Ideally such an algorithm should be computationally simple yet sufficiently accurate to yield results which approximate closely to actual system responses.

\subsection{The exponential input describing function}

If for a step input sing al to the non linear feedback system of figure 2 the output increases monotonically to a final steady state value, then the input to the non linear element will be of a monotonically decreasing form as show. This leads to the consideration of a model input signal to the non linearity which is an exponential time function (3). The exponential input describing function representation of the non linearity is determined by minimizing the integral-squared error in a linear approximation to the actual non linear output. The magnitude of this equivalent linear gain is thus determined as

$$
N_{E}=\int_{0}^{00} \frac{x(t) y\{x(t)\} d t}{x^{2}(t) d t} ; \text { For } x(t)=E e^{-t / T}
$$

This relationship generally results in a simple analytical expression for most common static non linearities and is thus easy to compute. In practice, of course, the input to the non linearity will be of the more general form

$$
\mathrm{E} \mathrm{e}^{-\mathrm{t} / \mathrm{T}} \cdot \operatorname{sine}\{\omega t+\Phi\}+\text { bias }
$$


where the harmonic content is determined by the systems dominant complex poles. However, if the percentage overshoot is constrained to $10 \%$ or less this harmonic component has little effect on the computed value of $N_{E^{*}}$ The effect of any bias level can be considered by a suitable adjustment to the value of $E$ used in the derivation of $\mathrm{N}_{\mathrm{E}}(4)$.

In this work the application of the exponential input describing function is limited to the study of single valued saturation type non linearities and all system linear elements are assumed to have essentially low pass frequency characteristics.

\subsection{Limit Cycle Prediction}

Sinusoidal input describing function techniques have been used widely in the study of limit cycle prediction in non linear single loop feedback systems where the application of complex frequency domain analysis has represented an intuitive extension of linear systems theory. Although the limitations of this approach are well documented, it has been shown recently (2) that the accuracy of Iimit cycle prediction can be improved by the addition of an error band to the $\hat{G}(j \omega)$ locus in the complex frequency comain. This error band is defined by a. set of error circles centred on the $\hat{G}(j \omega)$ locus which represent the contribution of higher harmonic signal components and this artifice allows the possibility of a more rigorous study of limit cycle behaviour than any treatment based only on a fundamental simusoidal analysis.

For the autonomous non linear feedback system of figure 3 where the single valued non linear element is constrained to a sector defined by lines with slopes $\alpha$ and $\boldsymbol{\beta}$ where $\boldsymbol{B}>\alpha$, the radius of an error circle on $\hat{G}(j \omega)$ corresponding to the $k$ harmonic is determined as

$$
\sigma(\omega)=\left\{\frac{\beta-\alpha}{2}\right\}^{2} /\left\{p(\omega)-\frac{\beta-\alpha}{2}\right\}
$$

where

$$
p(\omega)=\min _{\substack{k \neq 1 \\ k \text { odd }}}\left|\frac{\alpha+\beta}{2}+\hat{G}(j \omega)\right|
$$

and $P(\omega)$ is defined only for values of $\omega_{\text {on }}$ the set

$$
\Gamma \Delta \quad\left\{\omega: \rho(\omega)>\frac{\beta-\alpha}{2}\right\}
$$

The envelope of all such circles over a subset of $\Gamma^{\prime}$ of $\Gamma$ is the uncertainty band. The reason for choosing a subset is that as $\boldsymbol{\omega}$ decreases below a certain value, the error circles become increasingly large and cease to give useful information. In the case of a complete intersection of the uncertainty band with the describing function locus as in figure 4 it can be shown that limit cycle operations can be rigorously predicted with a frequency in the range given by the 
span $\boldsymbol{\omega}_{1}$ to $\boldsymbol{\omega}_{2}$ and an amplitude lying within the range between $x_{1}$ and $x_{2}$. In addition the closed loop system can have no periodic oscillation of least frequency $\omega \in \Gamma^{\prime}$ if there is no intersection of any part of the uncertainty band with the describing function locus. It should be noted that al though this algorithm adds a degree of mathematical rigour to the normal intuitive sinusoidal describing function approach, the results obtained will only be particularly useful if the harmonic content of the signal is restrained within reasonable bounds and the requirement of essentially low pass linear system elements still remains.

$\underline{3 .}$ Computational Procedures

The non linear system is assumed to have the structure shown in figure 5 where $K(s)$ represents a precompensator with linear elements. A general structure for the elements of $\mathrm{K}(\mathrm{s})$ must first be chosen. This can be as complex as desired but it is found useful to start with very simple forms such as a proportional plus integral element or an elementary phase lead structure. The magnitude of the input step signal is now chosen and the equivalent gain derived for the non linear element using the exponential input describing function approximation. The linearised model is now examined for closed loop stability and a set of coefficients automatically computed for the elements of $K$ to ensure this condition. For the chosen input step signal magnitude, the loop time response is now specified as a set of constraints which fix limits to such factors as rise time, percentage maximum overshoot and steady state error. Physical constraints can also be placed on the coefficients of the elements of $K$ and on the magnitude of the input forcing function from the compensator.

Starting from the initial values of the coefficients of the elements of $K$ which ensured closed loop stability for the linearised system, the design program now iterates to find suitable values for these elements which will meet the imposed constraints. At every stage in the iteration the stability of the linearised system is first checked and the derived values of the coefficients then used to seek for solutions of the harmonic balance equation in the complex frequency domain where the non linear element is now represented by its corresponding sinusoidal input describing function. Limit cycle operation is deemed to exist if, over a chosen frequency set $r$, error circles associated with either the third or fifth harmonic of the fundamental intersect the describing function locus. By incorporating this second computational procedure as a subroutine of the iteration sequence it is thus possible to ensure that each point in the admissable space represents a closed loop system which is theoretically free of limit cycle behaviour.

The computer program gives the result of each iteration so that the user is aware not only of the progress of the design but also of those particular specifications which are most difficult to meet within the limitations imposed by physical constraints and the nature of the compensator forms chosen for the design. The addition of a computer graphical facility allows system response to be studied 
in both the time and frequency domain at any stage in the design procedure if required.

4. An example of use

A precompensator is to be designed for the system shown in Figure 6 to ensure closed loop stability and a time response to a unit step signal which is defined by the following set of inequalities

$$
\begin{aligned}
& \text { Rise time }<6 \text { seconds } \\
& \text { settling time }<10 \text { seconds } \\
& \text { percentage overshoot }<10 \% \\
& \text { maximum compensat: or output }<10
\end{aligned}
$$

A simple compensator structure was chosen of the form

$$
\mathrm{K}(\mathrm{s})=\frac{\mathrm{K}_{1}\left(1+\mathrm{K}_{2} \mathrm{~s}\right)}{1+\mathrm{K}_{3} \mathrm{~s}}
$$

where each $\mathrm{K}$ was restricted to a value 0.1

An initial investigation for limit cycle operation in the uncompensated system produced the computer graphical display shown in figure 7 which predicts a limit cycle with a magnitude in the range $1.3 \rightarrow 1.6$ and frequency in the range $1.9 \rightarrow 2.05$ Radians/sec. The limit cycle subsequently obtained from an analogue simulation of the uncompensated system is shown in trace $d$ of figure 8 and has a measured magnitude of 1.43 and frequency 1.6 Radians/sec.

After five iterations of the design program a compensator of the form

$$
K(s)=\frac{0.08(1+.035 s)}{(1+0.0455 s)}
$$

was obtained

and the computed closed loop time response was as shown in trace $b^{1}$ of figure 8 . The corresponding analogue computer simulation result is shown in trace $b$ of figure 8. Traces $a$ and $\mathfrak{c}$ give the output response of the same simulated system with input step signal magnitudes of 0.5 and 2.0 respectively. The behaviour of the compensated system in the frequency domain is shown in the computer graphical display of figure 9 which indicates as expected, that there is now no intersection between the error circles on $\hat{\mathbf{G}}(\mathbf{j} \boldsymbol{\omega})$ and the describing function locus. The required design specifications have been achieved. Computation required approximately 30 seconds on the PDP 10 computer at U.M.I.S.T.

\section{Evaluation}

The successful extension of the method of inequalities to non linear feedback system design depends on the validity of the approximations used for 
representing the non linear element. Although simple in form, the exponential input describing function approximation has proved both remarkably accurate and robust in its prediction of indicial loop responses. Part of the reason for this must lie in the fact that while at the beginning of the iteration sequence the form of signal input to the non linear element may diverge appreciably from that assumed, as the computation proceeds the compensator structure enforces the required shape on the output time function which, in turn, increases the accuracy of the approximation and allows rapid convergence of the design. The convergence process can be further improved at little cost in computation time by calculating the magnitude of the input signals to the non linear element at each iteration and adjusting the values of the derived linear gains appropriately.

In theory, any derived compensator will meet the required performance in the time domain for the one input signal magnitude chosen at the beginning of the design. It is not difficult to recalculate the coefficients of the compensator elements over a range of magnitudes of input signal and derive a compromise set of coefficient values. In practice, over a very wide range of worked examples it has been found that provided the original input signal magnitude is chosen sensibly, the result produced by the exponential input describing function approximation is sufficiently robust to render this exercise generally unnecessary. This is illustrated in the example given.

Initially the non linear function was restricted to a simple saturation element as it was found that in the open loop condition the exponential input describing function approximation gave particularly good results with this type of non linearity. Within the context of the design program, however, equally good results have now been achieved with single valued non linear elements of different types and the range of application has thus been extended. The assumption of, essentially, low linear system elements still, of course, remains a vital prerequisite in any study involving the sinusoidal describing function philosophy. It has been recently shown (4) that the method can be extended to multi input, multi output, low pass linear systems containing a set of single valued, bounded non linear elements.

Within the method of inequalities the use of different algorithms than those discussed above is of course possible and these will be introduced as experience dictates to deal with particularly difficult system configurations which are not amenable to the simple approximations used here. It should be stressed that at every stage in the iteration process the user is faced with design decisions and the progress of the design and even its ultimate success will depend on a sensible evaluation of both initial constraints and possible controller structures. The user must also have some initial insight into those factors which influence the system under consideration and be aware at all times of the limitations inherent in the algorithms used in the computational procedure. 

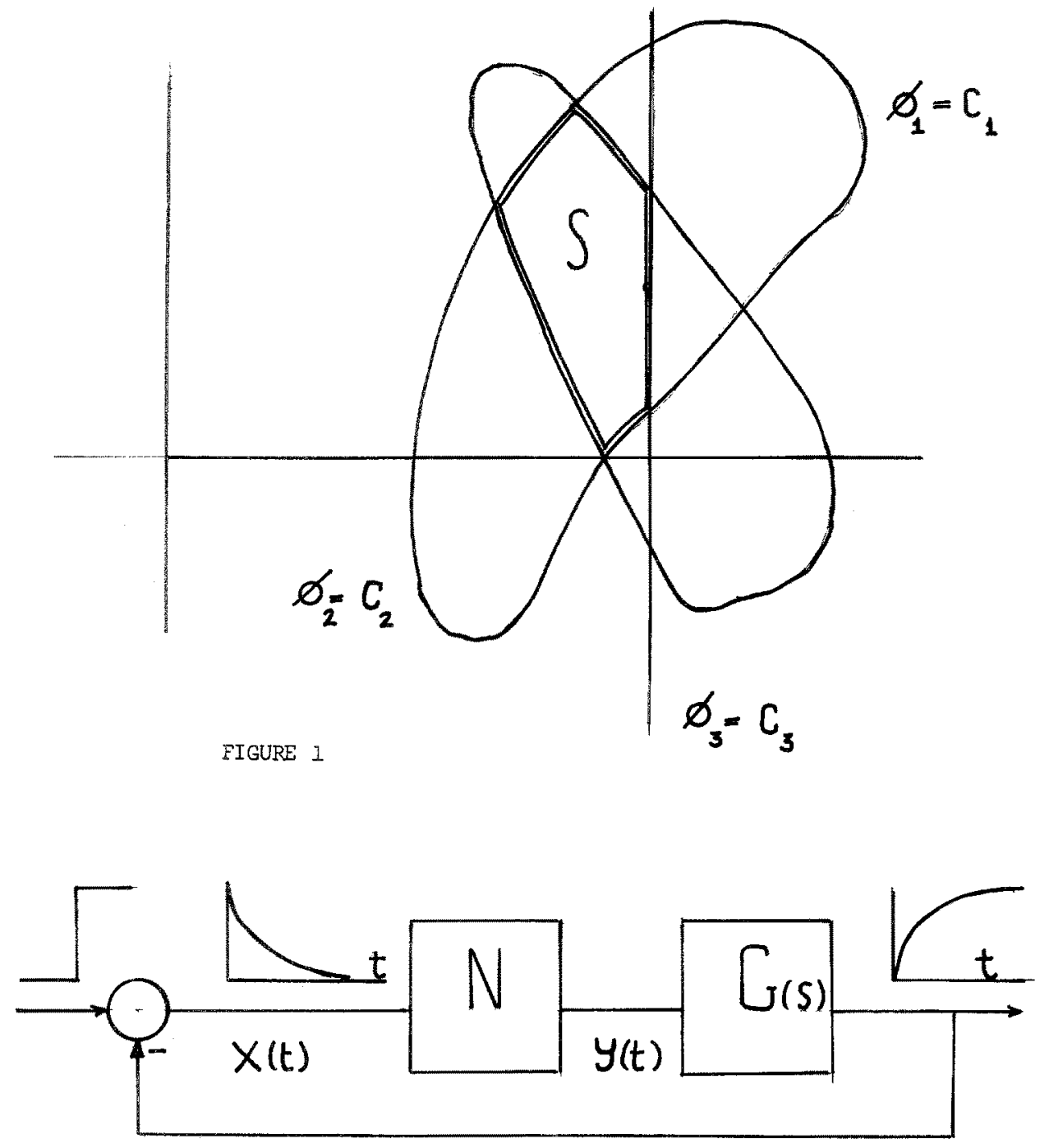

EIGURE 2

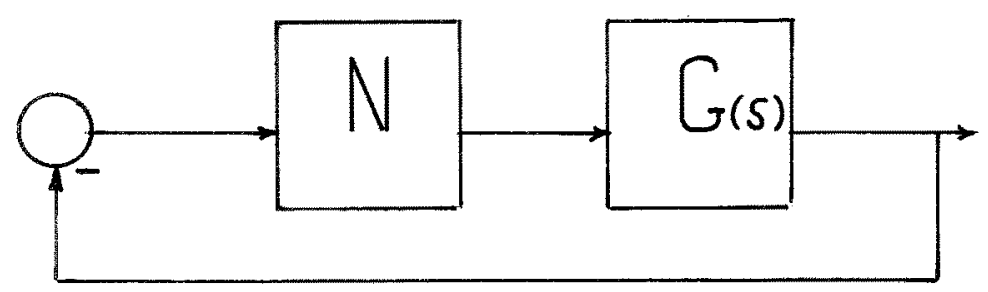

FIGURE 3 

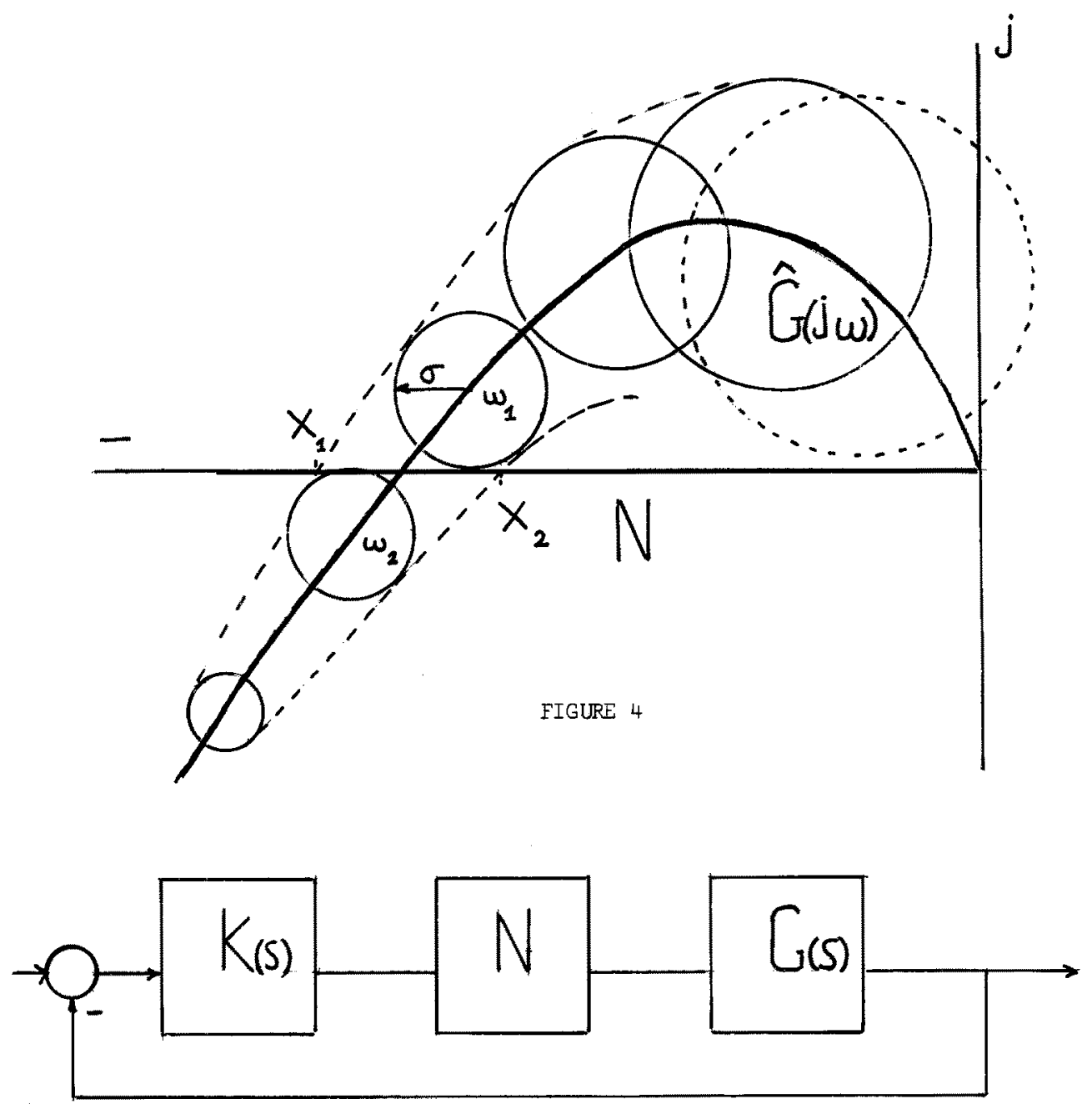

EIGURE 5

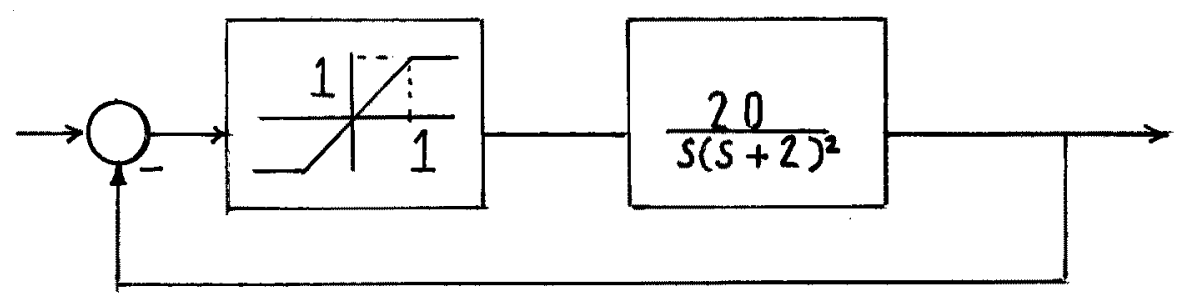

FIGURE 6 


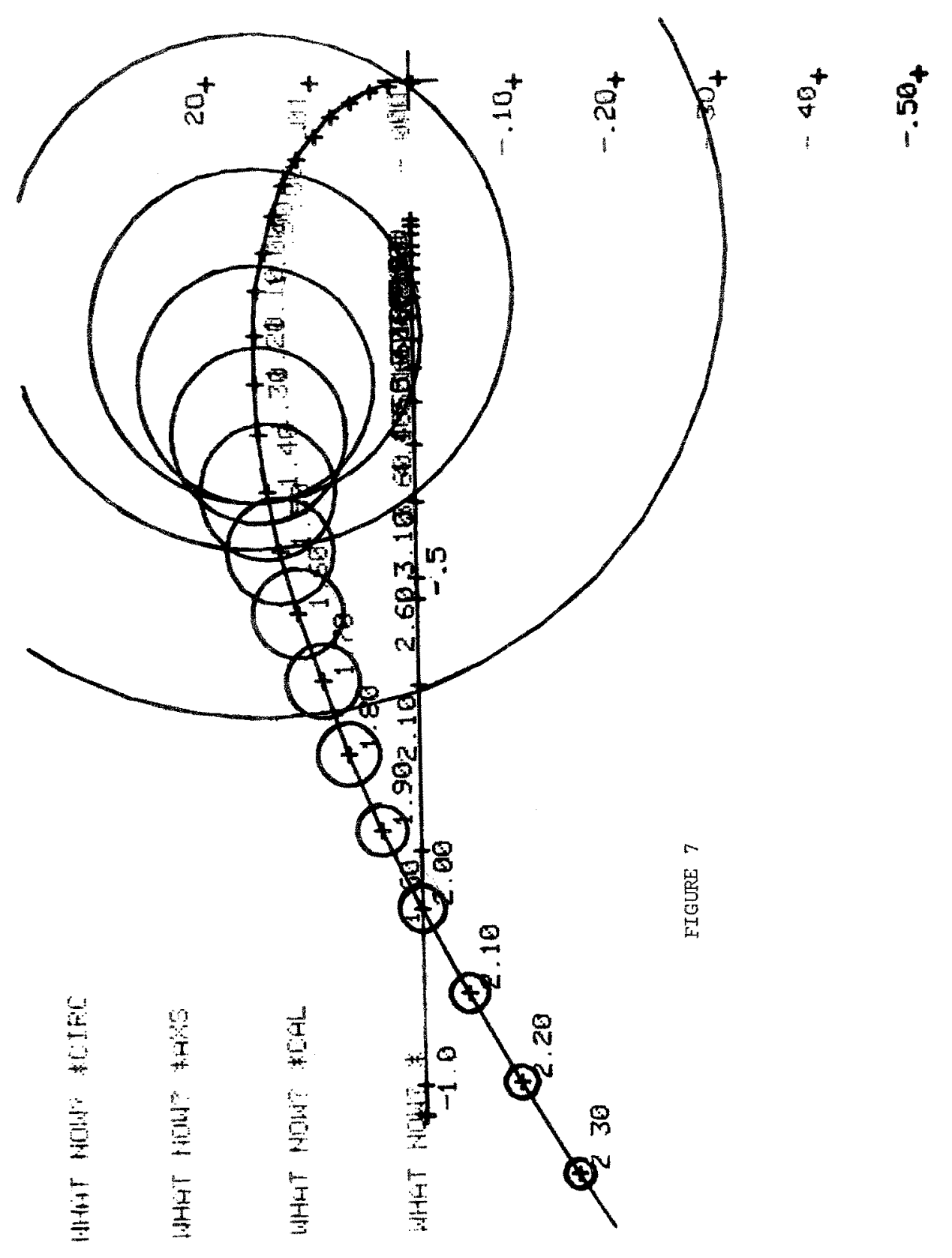




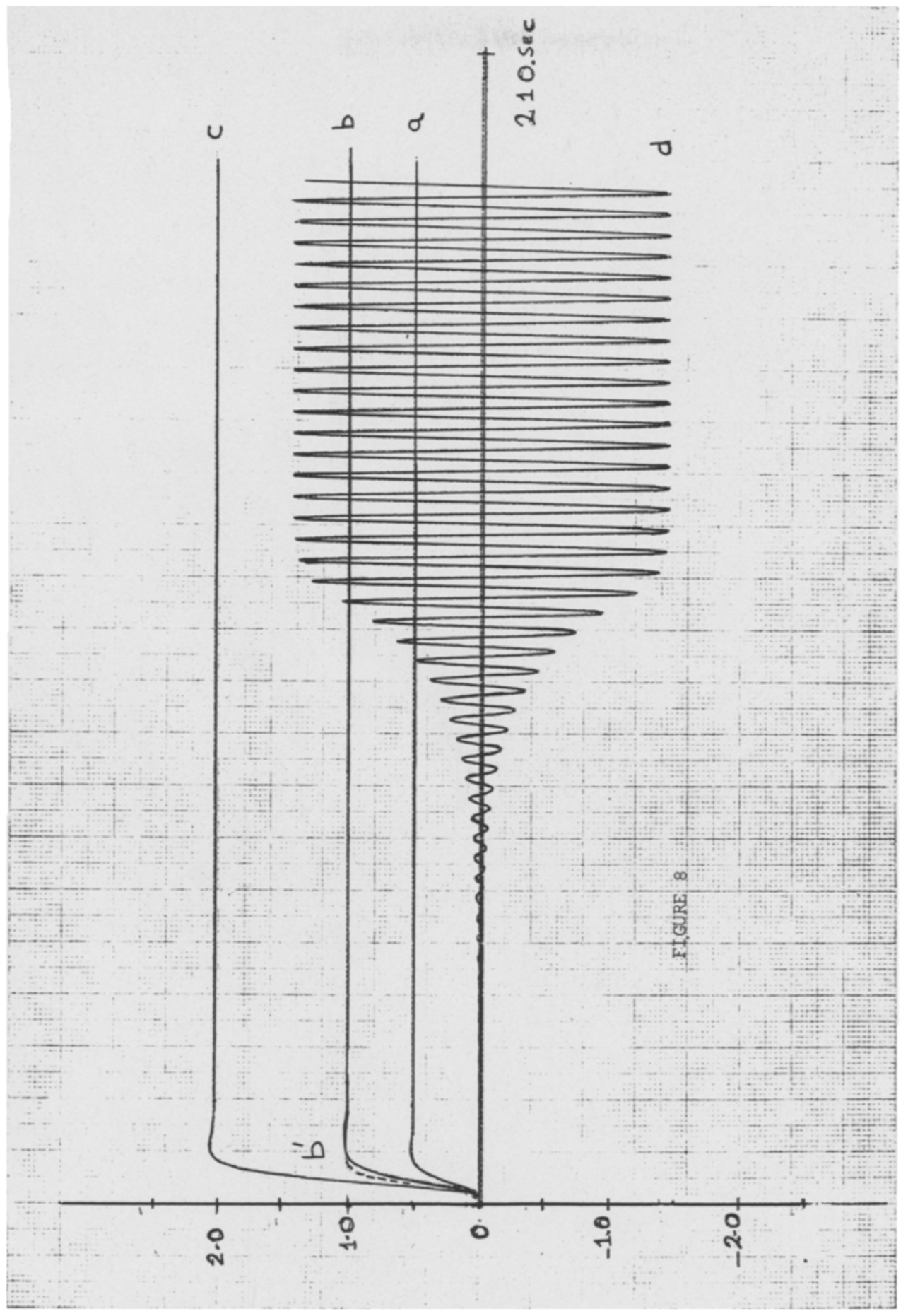


520

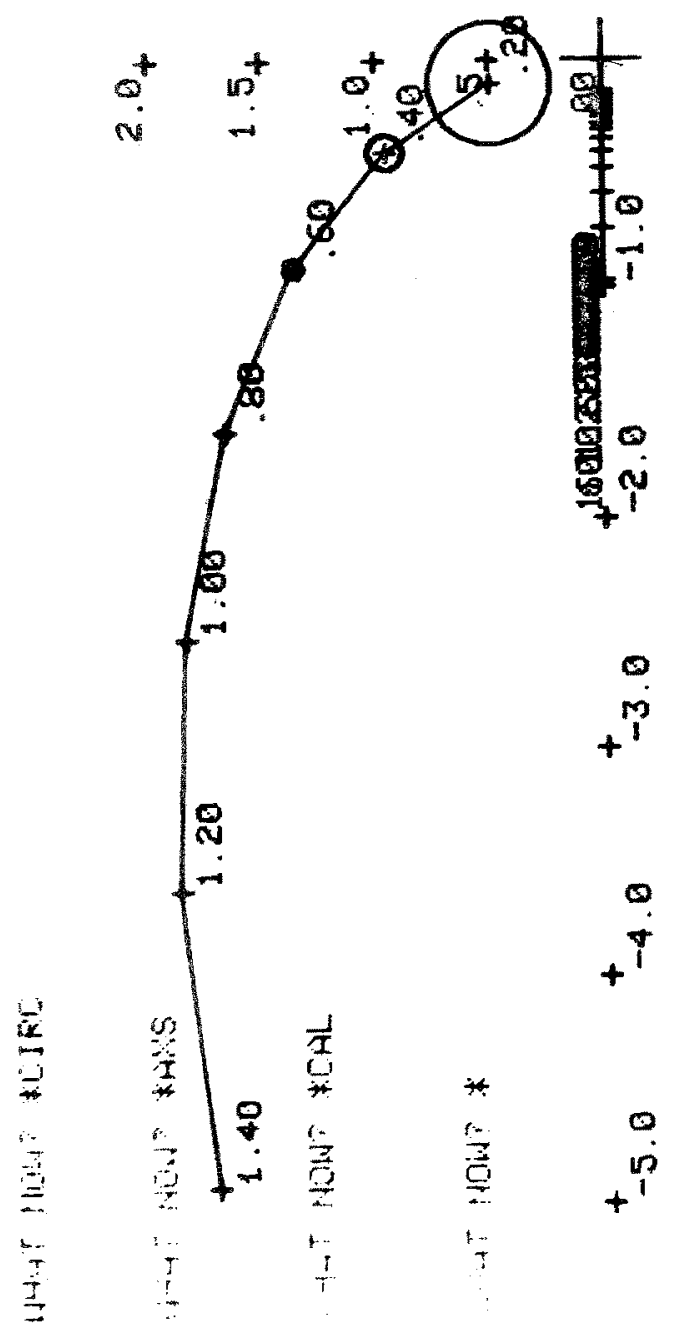

$\stackrel{9}{9}$ 


\section{References}

1. Zakian, V., AI Naib, U., "Design of dynamical and control systems by the method of inequalities". Proc. I.E.E. Vol. 120, No. 11. Nov. 1973. pp 1421-1427.

2. Mees, A., Bergen, A. R.; "Describing function revisited"; Proc. T.E.E.E. Vol. AC 20,1975 , pp $473-478$

3. Gelb, A., Vander Velde, W.; "Multiple input describing functions and non linear systems design". McGraw Hill 1958.

4. Al-Janabi, T. H.; "The numerical design of multivariable non linear control systems". Ph.D. Thesis, U.M.T.S.T., I975. 